\title{
New sulfonates and amides derived from berberine as Tdp1 inhibitors
}

\author{
Elizaveta Gladkova *, Arina Chepanova, Alexandra Zakharenko, Olga Luzina \\ ${ }^{1}$ N. N. Vorozhtsov Novosibirsk Institute of Organic Chemistry, Siberian Branch of the Russian Academy of \\ Sciences, 9, Akademika Lavrentieva Ave., Novosibirsk 630090, Russia; \\ 2 Novosibirsk State University, Pirogova str. 1, Novosibirsk 630090, Russia; \\ ${ }^{3}$ Novosibirsk Institute of Chemical Biology and Fundamental Medicine, Siberian Branch of the Russian \\ Academy of Sciences, 8, Akademika Lavrentieva Ave., Novosibirsk 630090, Russia;
}

Berberine $\mathbf{1}$ is known for its wide range of biological activity, including hypolipidemic, antibacterial, hypoglycemic, etc. Our interest is focused on the inhibitory properties of berberine and its derivatives in relation to the DNA repair enzyme Tdp1. Inhibition of various DNA repair enzymes, including Tdp1, is one of the promising fields in the evolution of antitumor therapy.

We synthesized and tested a number of berberine derivatives, which allowed us to identify leading compounds and understand which functional groups should contain a derivative and in which positions in order to exhibit inhibitory activity. According to SAR data analysis only tetrahydroberberine derivatives containing a reduced ring $C$ show inhibitory activity. The presence of substituents in both positions simultaneously (sulfonate group in 9 and bromine in 12) leads to increasing of inhibitory activity in comparison with unbrominated in 12 position analogues. Almost all of the most active derivatives exhibit low cytotoxicity. Thus, the results reveal that some berberine derivatives are potential agents that can be used in antitumor therapy.
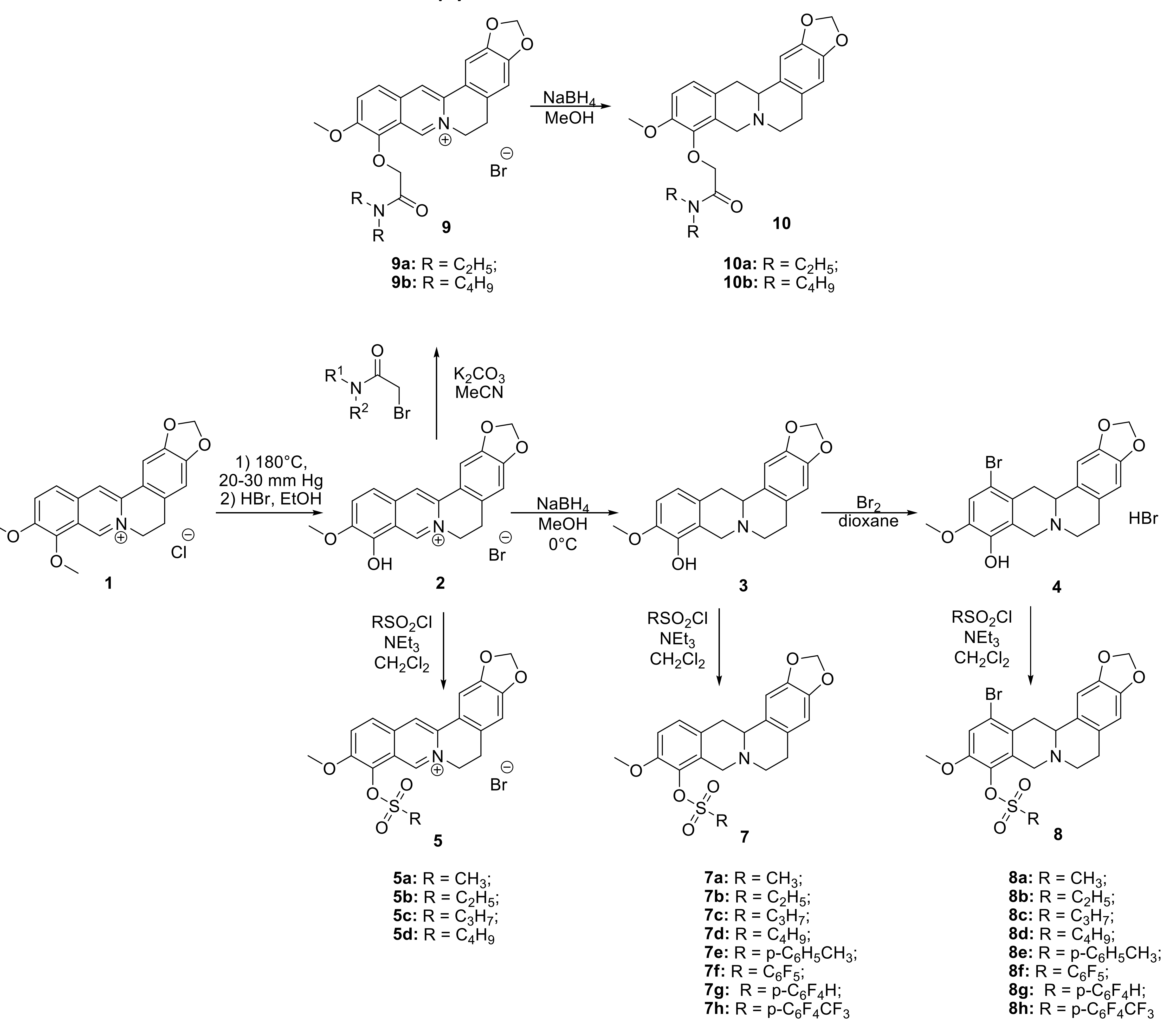

\section{Inhibitory activity}

Among the presented derivatives, the most significant inhibitory activity have shown compounds $\mathbf{7 f}$ - $\mathbf{7 h}$ and $\mathbf{8 f} \mathbf{- 8 h}$. Despite the fact that compound $\mathbf{8 f}$ shows the minimal IC50 value $(0.53 \mu \mathrm{M})$, the most promising derivatives are compounds $\mathbf{7 g}$ and $\mathbf{8 g}$ (IC50 $=$ 1.0 and $1.3 \mu \mathrm{M}$ correspondingly) due to their lower cytotoxicity in comparison with $\mathbf{8 f}$.

\section{Sensitizing effects}

The sensitizing effect of the berberine inhibitors on topotecan's cytotoxic potential was investigated. Their concentrations were varied with topotecan concentration of $2 \mu \mathrm{M}$, its $\mathrm{CC}_{50}$ for HeLa cells. Topotecan significantly increased the cytotoxicity of compounds $\mathbf{7 g}, \mathbf{8 g}$, and $\mathbf{7 f}$.

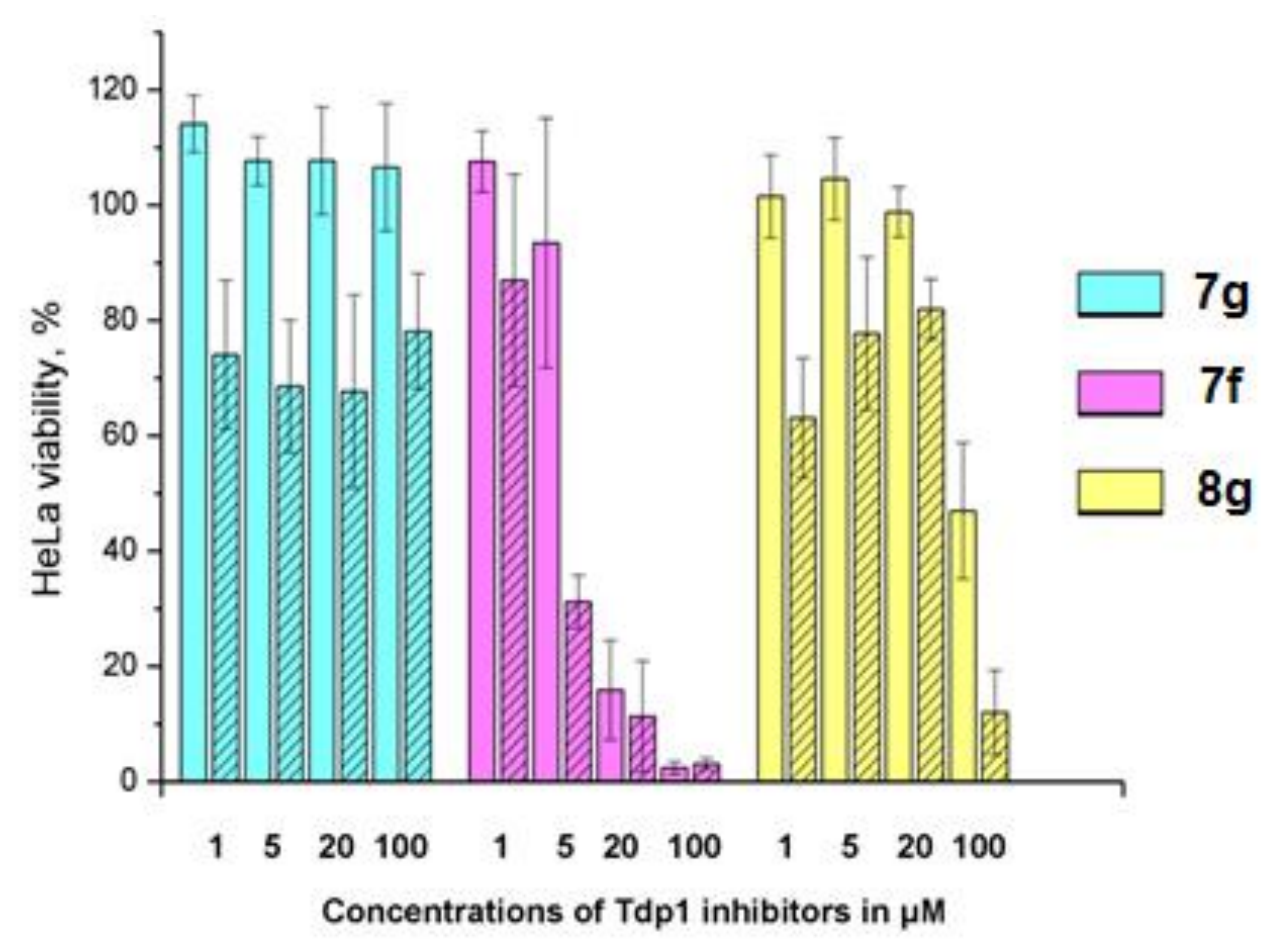

Non-toxic concentrations of the berberine derivatives $(5 \mu \mathrm{M})$ were then tested at different concentrations of topotecan. The most toxic compound $\mathbf{7 f}$ caused $20 \%$ cell death at this concentration; the rest of the compounds were not toxic. In general, our Tdp1 inhibitors doubled the cytotoxic potential of topotecan as can be seen in table.

\begin{tabular}{|c|c|c|}
\hline Compounds & $\mathrm{CC}_{50}, \mu \mathrm{M}-5 \mu \mathrm{M}$ Tdp1 inhibitor & $\mathrm{CC}_{50}, \mu \mathrm{M}-20 \mu \mathrm{M}$ Tdp1 inhibitor \\
\hline Tpc & \multicolumn{2}{|c|}{$6.8 \pm 1.1$} \\
\hline $\mathrm{Tpc}+7 \mathrm{~g}$ & $3.5 \pm 0.6$ & $2.3 \pm 0.5$ \\
\hline Tpc + 8g & $2.9 \pm 0.4$ & $3.3 \pm 1.1$ \\
\hline$T p c+7 f$ & $1.7 \pm 0.3$ & not determined \\
\hline
\end{tabular}

